\title{
Introduction : la Bourgogne : dynamiques spatiales et environnement
}

\section{Yves Boquet}

\section{(2) OpenEdition}

\section{Journals}

Édition électronique

URL : http://journals.openedition.org/rge/1193

DOI : $10.4000 /$ rge. 1193

ISSN : 2108-6478

Éditeur

Association des géographes de l'Est

Édition imprimée

Date de publication : 1 janvier 2006

ISSN : 0035-3213

Référence électronique

Yves Boquet, «Introduction : la Bourgogne : dynamiques spatiales et environnement », Revue

Géographique de l'Est [En ligne], vol. 46 / 1-2 | 2006, mis en ligne le 11 septembre 2008, consulté le 25 septembre 2020. URL : http://journals.openedition.org/rge/1193 ; DOI : https://doi.org/10.4000/rge. 1193

Ce document a été généré automatiquement le 25 septembre 2020

Tous droits réservés 


\title{
Introduction : la Bourgogne : dynamiques spatiales et environnement
}

\author{
Yves Boquet
}

1 Ce numéro de la Revue Géographique de l'Est est consacré à la Bourgogne. Dotée d'une forte identité patrimoniale par son histoire et ses vins, la Bourgogne est cependant soumise à des forces extérieures qui en menacent l'équilibre apparent. Les articles rassemblés dans ce numéro ont comme ambition de faire le point sur des dynamiques majeures actuellement à l'œuvre dans la région.

2 Jean-Jacques Bavoux manie les métaphores pour définir les modes de structuration du territoire bourguignon. Extraite de l'espace français et européen environnant, la Bourgogne pourrait s'apparenter à une île dont le centre géographique quasi-vide du Morvan s'oppose à des périphéries littorales bien peuplées, l'axe séquanien DijonMâcon étant vu comme une Riviera «sous le vent » tournant le dos aux autres pôles périphériques (Nevers, Auxerre). La Bourgogne, analysée ensuite dans un continuum spatial élargi, apparaît par contre comme un ensemble régional flou dont les marges sont en cours de "métamorphisme " au contact des puissantes agglomérations parisienne au Nord-Ouest (Sénonais) et lyonnaise au Sud-Est (Mâconnais), tandis que la «Bourgogne » nivernaise est bien plus ligérienne que connectée à Dijon. Une troisième analyse, réticulaire et non plus surfacique, fait apparaître la Bourgogne, espace «intermédiaire» typique, comme un isthme essentiel aux circulations transeuropéennes depuis des siècles, situation renforcée par les grands axes autoroutiers et ferroviaires. La situation centrale de la Bourgogne empêche paradoxalement l'émergence d'un pôle puissant, puisque la combinaison des simples transits et la fluctuation des nœuds de circulation bourguignons au gré des techniques de transport ont entraîné le développement d'une aire nodale polynucléaire allongée sur 200 km de Dijon à Lyon, sans que Dijon, Beaune, Chalon ni Mâcon puissent en tirer un bénéfice indiscutable pour asseoir leur prééminence dans une région où cet axe de circulation est très périphérique. Malgré les efforts des édiles régionaux pour attirer 
des équipements de transports et de logistique structurants, la Bourgogne apparaitt surtout comme un espace de transit.

3 Le réseau de lignes ferroviaires à grande vitesse, s'il traverse la Bourgogne et a permis la création de deux gares nouvelles, en périphérie des pôles urbains de Mâcon et le Creusot-Montchanin, n'a en effet pas eu d'impact majeur sur le dynamisme économique local à proximité de ces deux gares nouvelles. Première région française dotée de ces gares-bis, vite considérées comme des gardes "perdues» ou "du désert», la Bourgogne a été un laboratoire de cette expérience de gares exurbanisées allant à l'encontre des logiques habituelles de gares proches du centre-ville. Valérie Facchinetti-Mannone montre que ces gares, mettant des aires urbaines en difficulté comme celle du Creusot, à moins de deux heures de Paris, et à 30-40 minutes de Lyon, ont suscité des projets enthousiastes de zones d'activités économiques dont l'échec a été rapidement évident. En effet, ces gares sont peu desservies par les TGV, la plupart des trains ne s'y arrêtant pas, comme en gare TGV-Haute-Picardie entre Amiens et St Quentin. De plus, elles sont déconnectées du réseau classique et l'intermodalité avec les TER y est très malcommode, si ce n'est inexistante. Les stratégies ambitieuses de valorisation de la desserte à grande vitesse ont échoué, et Mâcon-TGV ou Le CreusotTGV sont en fait de simples haltes facilitant l'accès à Paris, sans créer de polarités nouvelles.

, capitale régionale, beaucoup mieux desservie par les rames TGV que les autres villes bourguignonnes, en attendant de devenir peut-être, un nouveau carrefour de lignes à grandes vitesse, avec la réalisation du TGV Rhin-Rhône à trois branches qui suscite actuellement débat local sur la localisation précise de la nouvelle gare TGV intra-urbaine, est sujette comme d'autres métropoles régionales à des dynamiques intenses d'expansion périurbaine.

5 André Larceneux examine les principales facettes de l'étalement urbain dijonnais, dont la nouvelle définition spatiale du pôle urbain est l'expression. Favorisé par les politiques de transports (construction de radiales rapides permettant d'habiter plus loin du pôle dijonnais) et du logement (aides à l'accession à la propriété), l'étalement urbain se développe malgré une concentration des emplois sur le pôle dijonnais. L'emploi tend en effet à diminuer dans les communes les plus périphériques et à augmenter dans les communes constituant le Grand Dijon (communauté d'agglomération). Aisément accessibles depuis Dijon, Is-sur-Tille au Nord et Nuits-StGeorges au Sud tendent à s'intégrer au pôle dijonnais. Les mouvements pendulaires au sein de l'aire dijonnaise ne cessent de s'accroître, posant aujourd'hui des problèmes croissants de congestion des axes routiers d'entrée dans la ville et contribuant à un accroissement des nuisances.

6 Cette croissance périurbaine implique aussi une pression accrue sur la ressource en eau. Non seulement l'expansion entraîne de coûteux travaux de raccordement aux réseaux existants et la recherche de nouveaux approvisionnements, mais la gestion de l'eau selon une logique de bassins-versants (trois en l'occurrence: Ouche, Tille et Vouge) et de nappes aquifères doit être confrontée à des structures intercommunales reposant sur des logiques différentes, car basées sur des critères socio-économiques et politiques et non "naturels » comme l'explique Emmanuelle Renaud-Hellier dans son examen des «territoires artificiels de l'eau » autour de Dijon. A l'heure des Schémas de Cohérence Territoriale, une meilleure articulation entre cadres territoriaux et systèmes d'action s'avère essentielle. 
7 Si la Bourgogne et l'aire urbaine dijonnaise peuvent être considérées comme des espaces aux marges floues, la logique des appellations d'origine contrôlée permet de définir précisément les communes viticoles de la région, à laquelle elles apportent une notoriété internationale. François Legouy, dans une approche statistique multivariée, analyse les données du recensement général de la viticulture de 2000 - complétées par les données de l'INAO - pour esquisser une typologie des communes viticoles de trois départements bourguignons (Yonne, Côte d'Or, Saône-et-Loire) et montrer qu'il existe bien un gradient viticole au sein de la viticulture de qualité, qui ne correspond que partiellement à la hiérarchie pyramidale des Appellations d'Origine Contrôlées bourguignonnes. Il distingue ainsi 7 grands types de communes viticoles en fonction de l'importance spatiale et économique de la filière viticole. Chablis, seule de la catégorie 7 (grands crus) dans l'Yonne et Meursault (au cœur de l'axe viticole des Grands Crus du talus côte d'orien au Sud de Dijon, émergent comme les communes rurales les plus fortement marquées par la viticulture.

8 Méconnus du grand public, les vignobles du Nivernais, de l'autre côté du Morvan par rapport aux vignobles prestigieux des Côtes de Nuits et de Beaune, après avoir décliné après 1890 puis quasiment disparu dans les années 1960, sont aujourd'hui en train de renaître. Marie-Pierre Cerveau montre comment, dans un contexte de concurrence exacerbée et de crise des vignobles français, les Coteaux du Giennois ou les Coteaux Charitois ont pu s'appuyer sur des structures d'encadrement locales et être reconnus par les organes viticoles officiels et les revues spécialisées. La détermination de quelques individus a été un élément déterminant pour cet essor, qui passe par Internet et des victoires en concours de dégustation.

9 La Bourgogne, carrefour de circulations, s'est retrouvée pendant l'été 2003 (bonne année pour les grands crus!) sous l'influence d'un anticyclone particulièrement immobile qui a été un facteur essentiel de la canicule meurtrière du début du mois d'août. Jean-Pierre Besancenot, Sabrina Havard et Etienne Cassagne reviennent sur cet épisode en examinant les facteurs de surmortalité liée à la canicule dans l'espace bourguignon, un des plus marqués par les fortes températures (39,8 $8^{\circ}$ à Mâcon, 39, $3^{\circ}$ à Dijon, $39,2^{\circ}$ à Nevers et $41,1^{\circ}$ à Auxerre qui a connu huit journées consécutives à plus de $40^{\circ}$ ). Aux fortes températures diurnes se sont ajoutées des «minima » nocturnes exceptionnellement élevés et éprouvants ( $26,3^{\circ}$ près de Beaune). L'air était cependant peu humide, ce qui a évité des conditions encore plus pénibles. Néanmoins, l'analyse des données de Météo-France permet de déceler des anomalies, Chalon-sur-Saône ayant été curieusement " épargnée " par les nuits « tropicales » tout comme Nevers. On a pu noter l'apparition d'ilots urbains de chaleur nocturne au cœur de la canicule alors que les campagnes étaient au moins aussi chaudes que les villes durant l'après-midi. L'impact sanitaire de la canicule a été inégal : maximale dans l'Yonne, la surmortalité à court-terme a été nettement plus faible dans la Nièvre. L'analyse de la mortalité à moyen terme (automne 2003-hiver 20004) indique que la surmortalité de l'été a précipité le décès de personnes fragiles, entraînant une sous-mortalité en février et mars, le bilan sur 8-10 mois étant équivalent aux valeurs habituelles. Les principales victimes de la canicule ont été des femmes âgées habitant en ville, avec une nette corrélation entre pics de surmortalité et nuits étouffantes. Les auteurs soulèvent en conclusion la question de la pertinence des systèmes d'alerte sanitaire en cas de canicule lorsque le nombre de stations météorologiques de référence est trop faible, comme dans le cas de la Saône-et-Loire. 
10 Yves Richard, Marie Laure Houzé et Serge Taboulot s'intéressent quant à eux aux techniques de prévision des pics d'ozone atmosphérique, enjeu de santé publique majeur comme l'a montré la période de canicule évoquée dans l'article précédent. Une collaboration entre les chercheurs climatologues de l'Université de Bourgogne, MétéoFrance et ATMOSF'air a permis de tester une méthode statistico-dynamique de prévision locale des concentrations d'ozone, dont les auteurs présentent la méthodologie et évaluent la pertinence au travers de l'épisode caniculaire exceptionnel de l'été 2003.

11 Ces quelques articles sont pour la plupart l'expression des travaux de recherche de géographes dijonnais, également engagés dans des études à base bourguignonne sur les conflits entre périurbanisation et viticulture au Sud de Dijon, la temporalité des mobilités urbaines, les représentations numériques de la ville, les futures gares du TGV Rhin-Rhône, la gestion des déchets en zone périfrancilienne.

Dans le cadre de la mise en place du LMD, les deux laboratoires de géographie de l'Université de Bourgogne (Théma-Dijon et CRC-centre de recherches en Climatologie) ont démarré en 2004 un master professionnel «Transports Mobilités Environnement " et en 2005 une licence professionnelle "Aménagement du Paysage, Gestion du Patrimoine Végétal en Milieu Urbanisé » a été ouverte, tandis que dans le cadre de la MSH de Dijon une équipe pluridisciplinaire, en liaison avec l'Institut Universitaire de la Vigne et du Vin Jules Guyot, se penche sur le patrimoine viticole régional.

Autant de thèmes qui pourront apparaître sous formes d'articles dans un futur numéro de la Revue Géographique de l'Est.

\section{AUTEUR}

\section{YVES BOQUET}

Professeur à l'université de Bourgogne, Directeur du département de géographie 